\title{
Appunti intorno al classicismo rinascimentale: Sannazaro e i latini nelle Rime di Pietro Bembo
}

\author{
Amelia JURI \\ Università di Losanna
}

\begin{abstract}
Il saggio intende mostrare la presenza della poesia quattrocentesca nelle Rime di Pietro Bembo, al fine di restituire un'immagine più precisa del suo classicismo e più in generale del suo esercizio poetico. Si prendono quindi in esame alcuni testi in cui Bembo dialoga strettamente con Sannazaro e la tradizione classica, che testimoniano da una parte la falsità della presunta ortodossia petrarchesca di Bembo, dall'altra il rilievo assunto dai temi nel processo imitativo.

Keywords: Pietro Bembo, Quattrocento, Iacopo Sannazaro, imitazione, lirica petrarchista
\end{abstract}

Nella sua nota recensione Per una storia della lingua italiana Carlo Dionisotti affermava che «la vittoria di questa tesi [scil. del Bembo] portò a una sommaria esecuzione del Quattrocento latino e volgare», e che solo «nel tardo Cinquecento e nel primo Seicento si ebbe un singolare, ingente recupero della tradizione volgare e di quella latina, cosí umanistica come scolastica, del Quattrocento, e di quel che il Quattrocento aveva ereditato dal passato e fatto proprio».' Questo giudizio, insieme a quelli di altri importanti studiosi, ha avuto delle ricadute negative negli studi sul petrarchismo cinquecentesco, schiacciando nello specifico le rime bembiane sulle posizioni del grammatico, autore delle Prose, e provocando una riduzione del classicismo cinquecentesco a un'imitazione esclusiva di Petrarca. È così mancata finora la preoccupazione di verificare la coincidenza o lo scollamento tra le Rime e quanto viene sostenuto nel trattato, ma soprattutto di sondare altri aspetti non meno importanti di quello linguistico, prevalente nelle Prose (ci si riferisce in particolare ai debiti verso la tradizione classica e più recente, quattrocentesca). Si è poi sommata a questo fattore la tendenza a intendere il petrarchismo come la scomposizione del modello in frammenti svincolati dal contesto originario e dai significati di cui quest'ultimo era portatore. $\mathrm{Ne}$ è derivata (o quantomeno ne è inscindibile) una pressoché totale noncuranza dei temi, la quale ha prodotto a sua volta notevoli fraintendimenti e ha avuto spesso come esito la trasformazione delle poesie di Bembo in una collana (centonaria) di schegge petrarchesche. ${ }^{2}$ Nei commenti l'attenzione

I Carlo Dionisotti, Per una storia della lingua italiana, in Id., Geografia e storia della letteratura italiana, Torino, Einaudi, 1967, pp. 89-124, a p. II7.

2 Basti ricordare qui le parole di Gardini, che pure ha contribuito a una migliore compren- 
verte perlopiù sulle coincidenze lessicali e sintagmatiche con i Fragmenta, mentre ne vengono trascurate altre di natura tematica che invero informano i testi sia dal punto di vista macroscopico (retorico-inventivo) sia in una prospettiva microscopica (lessicale), non di rado risultando più pertinenti anche per certi riscontri puntuali con il Canzoniere. L'apporto maggiore in questo senso viene dalla tradizione quattrocentesca (innanzitutto Giusto e Sannazaro) e latina, attraverso la quale Bembo filtra l'esperienza petrarchesca, o cui attinge in modo diretto, dando luogo a stratificazioni intertestuali molto dense ed elaborate.

Riconoscere questo rapporto vitale con il Quattrocento e la classicità non significa negare la sostanziale differenza tra il petrarchismo bembiano, cinquecentesco, e quello del secolo precedente, bensì porre rimedio al processo di atrofizzazione cui sono stati sottoposti i testi di Bembo nei secoli, e maturare un' idea più complessa del suo classicismo, il quale è radicato nella sua formazione umanistica ed è sì fondato sull'individuazione di Petrarca come modello unico in sede teorica, ma implica altresì la comprensione dei meccanismi profondi di quella lirica, incluse le sue dinamiche imitative. ${ }^{3}$

sione dell'imitatio di Petrarca: «La grandezza del poeta ora dipende dalla capacità con cui egli dimostra di piegare i limitati segni di un vocabolario lirico dato a variazioni e ricombinazioni infinite. [...]. La lingua di Petrarca è, così, ridotta a un insieme preconfezionato di semantemi potenziali, di significanti puri, ambigui [...], i cui significati siano determinati dalla collocazione nel nuovo messaggio. [...]. È come se Bembo perseguisse il sogno utopico di ritrascrivere il Canzoniere in un'identica forma, cambiando però il referente di ogni segno» (Nicola Gardini, Bembo: un codice volgare per la lirica moderna, in Id., Le umane parole. L'imitazione nella lirica europea del Rinascimento da Bembo a Ben Jonson, Milano, Mondadori, 1997, pp. 5I-94, a pp. 73 e 87). Oppure la famosa definizione di Quondam del petrarchismo come «sistema linguistico della ripetizione» in cui le «possibilità comunicative si giocano tutte sul piano dell'elocutio, essendo inventio e dispositio predefinite dal codice» (Amedeo Quondam, Il naso di Laura. Lingua e poesia lirica nella tradizione del Classicismo, Modena, Panini, 199I, p. 193). Di recente forti impulsi a una rivalutazione della poesia bembiana, specie nell'ambito delle fonti latine, sono stati dati dall'edizione del commento di Quattromani (Pietro Petteruti Pellegrino, Quattromani lettore di Bembo. Studio e edizione dei Luoghi delle rime, tesi di dottorato, Università di Losanna, 2015; ma vd. anche Guglielmo Gorni, Un commento inedito alle Rime del Bembo da attribuire a Sertorio Quattromani, in «Schifanoia», XV-XVI, I995, pp. I2I-I32, e Pietro Bembo, Rime, a cura di Guglielmo Gorni, in Poeti del Cinquecento, I, a cura di G.G., Massimo Danzi e Silvia Longhi, Milano-Napoli, Ricciardi, 200I, pp. 5I-I89), e da Simone Albonico, Appunti su "forma" e "materia" nella poesia di Pietro Bembo e del suo tempo, in Lirica in Italia I494-I530. Esperienze ecdotiche e profili storiografici, a cura di Uberto Motta e Giacomo Vagni, Bologna, Emil, in corso di pubblicazione (e Id., Autour de "forma" et "materia" dans la poésie de Pietro Bembo et de ses contemporains, «Italique», XIX, 20I6, pp. 303-332.

3 Negli ultimi anni alcuni studiosi hanno finalmente intrapreso delle indagini volte a mostrare la varietà del petrarchismo cinquecentesco, nondimeno si sono in genere fermati a un discorso generale e astratto, privo di affondi testuali; vd. almeno Giorgio Forni, Pluralità del petrarchismo, Ospedaletto, Pacini, 20II; Roberto Gigliucci, Antipetrarchismo interno o petrarchismo plurale?, in Autorità, modelli e antimodelli nella cultura artistica e letteraria tra Riforma e Controriforma, Atti del Seminario internazionale di studi (Urbino-Sassocorvaro, 9-II novembre 2006), a cura di Antonio Corsaro, Harald Hendrix e Paolo Procaccioli, Manziana, Vecchiarelli, 2007, 
Bembo colse senz'altro la profondità classica dei Fragmenta, come i suoi rapporti con la tradizione provenzale e le recenti esperienze italiane (in primis Dante), e su questa consapevolezza edificò la propria poesia, che non può dunque essere ridotta a una sterile imitazione (formale) del canzoniere petrarchesco.

D’altronde è lo stesso Bembo ad autorizzare un simile comportamento nei propri scritti, come ha ricordato Andrea Donnini:

una volta stabilita in sede teorica la sua esclusività [scil. della lezione stilistica di Petrarca], [Bembo] mantiene comunque uno spazio di rilievo per la propria libertà compositiva. Nell'epistola De imitatione fonda tale libertà distinguendo tra imitari e sumere. Dato un modello unico di stile, è consentito rivolgersi ad altri autori per prendere motivi, argomentazioni, contenuti, la cui espressione verrà ricondotta ai principi di riferimento: [...]. I prelievi devono essere condotti con parsimonia (parce [...] et prudenter): essenzialmente perché «praeclarius est illa omnia invenire nos, et quasi parere, quam ab aliis inventa mutuari». Il fine superiore è il miglioramento, l'emulazione: «ut quae mutuati sumus ipsi, ea splendidiora illustrioraque nostris in scriptis, quam in eius, a suo sumimus, conspiciantur: ut non minor in exornando laus, quam in inveniendo fuisse videatur». [...]. Se l'imitatio è riferita a Petrarca, il sumere si rivolge alla tradizione illustre della letteratura, volgare e latina. ${ }^{4}$

Senonché lo stile è quasi sempre inestricabile dai temi, sì che la struttura retorico-sintattica dei testi e in generale la loro forma sono normalmente condizionate da quelle del modello, le quali semmai vengono aggiornate. ${ }^{5}$ Inoltre va riconosciuto che il monito del "parce [...] et prudenter» dell'epistola latina non rispecchia del tutto la realtà, giacché la presenza dei classici latini e della lirica quattrocentesca è davvero ingente nelle Rime.

Un testo rappresentativo di questa funzione della tradizione extra-petrarchesca è la canzone Se 'l pensier che mingombra (8I; Asolani II XXVIII), in cui sono combinate le canzoni sorelle $R v f$ I25 e I26 con evidente intento emulati-

pp. 9I-IO2; Id., Appunti sul petrarchismo plurale, in «Italianistica», XXXIV, 2, 2005, pp. 7I-76; Stefano Jossa e Simona Mammana, Petrarchismo e petrarchismi. Forme, ideologia, identità di un sistema, in Nel libro di Laura. Petrarcas Liebesgedichte in der Renaissance, hrsg. von Luigi Collarile und Daniele Maira, Basel, Schwabe, 2004, pp. 90-II6.

4 Pietro Bembo, Le Rime, a cura di Andrea Donnini, Roma, Salerno, 2008, I, pp. LXXXI e LXXXV.

5 Il caso più eclatante è il sonetto 20 delle Rime, uno tra i più fortunati nel corso del Cinquecento. A questo riguardo ci si permette di rinviare a un proprio contributo in corso di pubblicazione: Sintassi e imitazione nei sonetti di Pietro Bembo, in Otto studi sul sonetto. Dai Siciliani al Manierismo, a cura di Arnaldo Soldani e Laura Facini, Padova, libreriauniversitaria.it edizioni, 20I7; nel quale si compiono altri rilievi circa il ruolo di Giusto de' Conti (su cui vd. Simone Albonico, Nota su Foscolo e Petrarca, in «Stilistica e metrica italiana», I, 200I, pp. 228-39, ripresa ora in Id., Autour de "forma" et "materia", cit.). 
vo, come hanno sottolineato Dionisotti e Claudia Berra. ${ }^{6}$ Ai rilievi metrici e argomentativi compiuti da questi ultimi si può forse aggiungere l'allusione a un testo classico che avvalora questa interpretazione dell'operazione bembiana. La conclusione della prima stanza (vv. IO-I5):

io che fra gli altri sono

quasi augello di selva oscuro humile, andrei, cigno gentile,

poggiando per lo ciel, canoro et bianco, et fora il mio bel nido

di più famoso e honorato grido.

condensa e rielabora l'ultimo carme del secondo libro delle Odi di Orazio:

Non usitata nec tenui ferar
penna biformis per liquidum aethera
vates, neque in terris morabor
longius invidiaque maior
urbes relinquam. Non ego pauperum
sanguis paternum, non ego, quem vocas,
dilecte Maecenas, obibo
nec Stygia cohibebor unda.
Iam iam residunt cruribus asperae
pelles et album mutor in alitem
superne nascunturque leves
per digitos umerosque plumae.
Iam Daedaleo notior Icaro
visam gementis litora Bosphori
Syrtisque Gaelatus canorus
ales Hyperboreosque campos.

Come Orazio aveva descritto la propria metamorfosi in cigno da uomo di estrazione umile, e quindi il conseguimento di una fama (di poeta) maggiore di quella di Icaro, così Bembo ipotizza che si trasformerebbe da uccello selvatico e umile in cigno qualora il pensiero riuscisse a restituire in versi la bellezza dell'amata, con un'estensione, dunque, del procedimento metaforico e il passaggio dalla certezza del futuro all'eventualità di un condizionale che prelude a un esito negativo (vv. I6-I8, «Ma non eran le stelle, / [...] / [...] disposte a tanto alzarmi»). La concordanza tematica garantisce in assenza di altri riscontri significativi nella tradizione la pertinenza delle singole coincidenze: la sintesi in una dittologia di album (alitem) e canorus, l'ampliamento

6 Pietro Bembo, Prose e Rime, a cura di Carlo Dionisotti, Torino, UTET, 1960, e Claudia Berra, La scrittura degli «Asolani» di Pietro Bembo, Firenze, La Nuova Italia, I996, p. 259. 
di notior in "più famoso et honorato", al contempo privato del termine di paragone mitologico, il passaggio dal denotativo «ego pauperum / sanguis paternum» al metaforico «augello di selva oscuro humile», dove vi è pure uno slittamento semantico (Bembo non allude alla propria condizione sociale, bensì alle proprie qualità poetiche) ${ }^{7}$

Questo discorso, qui per forza molto sommario, non vale solo per le rime di Bembo, ma per la maggior parte dei poeti cinquecenteschi, quantomeno per i migliori. Il rapporto con il Quattrocento (e i classici latini) è stato valorizzato dai commenti di Luca Milite alle Rime di Berardino Rota e di Stefano Carrai a Della Casa, nonché da Massimo Malinverni con particolare riguardo per la poesia cortigiana; ciononostante manca ancora uno studio sistematico. ${ }^{8}$ Gli studi sulla poesia cinquecentesca hanno infatti da tempo posto in evidenza l'importanza del Quattrocento nella produzione poetica più tarda, specie in ambito napoletano, ma l'hanno qualificata come una riscoperta successiva alla supposta censura bembiana. Sebbene in questa sede non si potrà fornire una dimostrazione completa, si auspica che la scelta dell'esperienza bembiana, dal forte valore paradigmatico, e l'analisi di alcuni testi incentrata sui contatti con uno dei maggiori lirici quattrocenteschi, Sannazaro, possano fornire una base, ancorché minima, per un ripensamento delle dinamiche imitative nella poesia cinquecentesca e del modo in cui Bembo concepiva l'esercizio poetico. Particolare attenzione verrà prestata pure alla costanza di questi legami, che percorrono in effetti tutta la produzione poetica del cardinale, dalle prime prove cortigiane fino agli ultimi esercizi.

Lo testimonia bene l'attacco del sonetto 73 delle Rime, indirizzato a Gasparro degli Obizzi e con ogni verosimiglianza non di molto anteriore al I530. ${ }^{9}$

Né tigre sé vedendo orbata et sola corre sì leve dietro al caro pegno,

7 I commenti cinquecenteschi a Orazio intendono univocamente l'«album [...] alitem» come un cigno, e interpretano il testo come un'allegoria della gloria poetica dell'autore, destinata a durare dopo la sua morte malgrado il suo rango sociale.

8 Berardino Rota, Rime, a cura di Luca Milite, Milano-Parma, Fondazione Pietro Bembo-Guanda, 200o; Giovanni Della Casa, Rime, a cura di Stefano Carrai, Torino, Einaudi, 2003; Massimo Malinverni, Paride in giudizio. Presenze quattrocentesche in un'ottava ariostesca (ed oltre), in «Rivista della letteratura italiana», IX, I-2, I99I, pp. I07-II8; Id., La lirica volgare padana tra Boiardo e Ariosto: appunti su una transizione rimossa, in Boiardo e il mondo estense nel Quattrocento, Atti del Convegno internazionale di studi (Scandiano-Modena-Reggio Emilia-Ferrara, I3-I7 settembre 1994), II, a cura di Giuseppe Anceschi e Tina Matarrese, Padova, Antenore, 1998, pp. 695-722. Importante pure Stefano Carrai, Il classicismo dell'Ariosto lirico, in Id., L'usignolo del Bembo. Un'idea della lirica italiana del Rinascimento, Roma, Carocci, 2006, pp. 85-99, quantunque il caso ariostesco sia in parte diverso.

9 Per tutte le datazioni dei testi si rinvia ai cappelli dell'edizione a cura di Donnini. 
né d'arco stral va sí veloce al segno, come la nostra vita al suo fin vola.

Le note di Donnini rimandano in modo non del tutto cogente a $R v f 285$ I-5 per la struttura retorica, sfugge infatti al commentatore un precedente quattrocentesco più pertinente tanto dal punto di vista retorico quanto da quello contenutistico. Si tratta della prima stanza della sestina 94 dei Sonetti e canzoni di Sannazaro, costruita in modo analogo su un paragone tra il movimento veloce di alcuni elementi naturali e la fugacità della vita umana.

Non fu cervo mai sì veloce al corso né leopardo o tigre in alcun bosco, né fiume aitato da continua pioggia, né nube che si affretti inanzi al vento, né vola sì leggier dardo né strale, come questa caduca e breve vita. ${ }^{\circ}$

Lo schema inventivo è identico, benché Bembo condensi la comparazione riducendo i termini di paragone. Tuttavia, dopo aver selezionato le immagini della tigre e della freccia, egli compie un passo ulteriore intessendo la comparazione di allusioni classiche. ${ }^{\text {II }}$ Donnini rinvia opportunatamente a Seneca, Med. 862-65, «huc fer pedes et illuc, / ut tigris orba natis / cursu furente lustrat / Gangeticum nemus», e Ovidio, Met. XIII 547-48, «Utque furit catulo lactente orbata leaena, / Signaque nancta pedum sequitur, quem non videt, hostem» per i vv. I-2, e Lorenzo de' Medici, Corinto 94, «come chi drizza stral veloce al segno» per il v. 3. Ad essi si possono però aggiungere altri riscontri, forse più stringenti: Giovenale, Sat. VI 270, «tum gravis illa viro, tunc orba tigride peior» e Lucano, Phars. V 403-6, «inde rapit cursus [...] / [...] / ocior et caeli flammis et tigride / feta» per l'immagine della tigre, nonché Stazio, Theb. VI 595-99, "volucres isdem modo tardius arvis / isse videntur equi; credas e plebe Cydonum / Parthorumque fuga totidem exiluisse sagittas. / Non aliter, celeres Hyrcana per avia cervi / cum procul impasti fremitum accepere leonis», dove compaiono il dardo e il cervo che tenta di fuggire da un leone (reimpiegati da Sannazaro). ${ }^{12}$ Per quanto riguarda i primi due versi

Io La sestina va a stampa in concomitanza con le Rime bembiane nel 1530, nondimeno, poste la vasta circolazione manoscritta delle rime sannazariane già a inizio secolo, nonché la forte somiglianza tra i due testi, non è affatto implausibile ipotizzare una lettura del testo anteriore alla princeps. L'edizione di riferimento qui e di seguito è Iacobo Sannazaro, Opere volgari, a cura di Alfredo Mauro, Bari, Laterza, I96I.

II Una costruzione non troppo dissimile ma in un contesto differente si trova in Boiardo, Amorum libri II I3 I-3.

12 In questo tessuto classico le uniche tracce petrarchesche sono «leve», che ricalca $R v f 574$, «et poi al partir son più levi che tigre», e il preziosismo in clausola («caro pegno»), mutuato da Rvf 2957. 
vi è dunque una specificazione rispetto al modello sannazariano: non più una semplice tigre che corre, bensì una tigre che cerca il cucciolo di cui è stata privata; mentre in relazione ai brani latini vi è un riorientamento delle similitudini, poiché i figurati erano da una parte il dolore e la rabbia di chi ha perso una persona cara (Seneca, Ovidio), dall'altra scene belliche di fuga o avanzata (Lucano e Stazio), che paiono dunque più vicine al brano bembiano. ${ }^{13}$ La quartina è quindi conclusa da un verso che combina due luoghi petrarcheschi, già segnalati da Donnini: Rvf 3154 , «ove scende vita ch'al fin cade», e 323 55, «ogni cosa al fin vola».

Simili tangenze sono altamente istruttive in quanto permettono di dedurre importanti corollari metodologici e critici: esse suggeriscono infatti un diverso - per non dire opposto - modo di concepire il rapporto contenuto-forma rispetto a quello che emerge dagli studi sul petrarchismo, in quanto il contatto con Sannazaro indica in modo indiscutibile la priorità dei temi sugli aspetti formali nella mente dei lirici cinquecenteschi, o meglio la loro interdipendenza. ${ }^{\mathrm{I}}$ Sembra del tutto inverosimile supporre che Bembo abbia pensato prima alla struttura retorica (già petrarchesca) poi al tema, è invece plausibile che egli abbia pensato dapprima al tema della caducità della vita e in seguito al modello (formale) offerto da Sannazaro. È dunque in parte inappropriata l'attitudine invalsa nella critica e nei commenti a segnalare quasi esclusivamente concordanze lessicali e sintagmatiche, e in senso più ampio coincidenze tra strutture linguistiche e/o retorico-sintattiche: queste ultime sono senz'altro significative, ma possono essere fuorvianti qualora la ricerca si limiti al capostipite Petrarca e non implichi una verifica di eventuali tangenze con altri autori in cui si sovrappone all'identità formale la concordanza tematica. Pare infatti urgente recuperare una visione e una valutazione dei testi più completa e rispettosa degli intenti degli autori.

Una diversa modalità di appropriazione della lirica sannazariana è presente in uno dei sonetti del sogno amoroso (IOI), incentrato sul motivo topico dell'apparizione della donna. Per la prima quartina

Giaceami stanco, e 'l fin de la mia vita venía, né potea molto esser lontano quando pietosa in atto honesto et piano madonna apparve a l'alma, et diemmi aita.

Gorni, ${ }^{15}$ seguito da Donnini, rinvia a Rvf 34I 3-5:

I3 Per inciso va registrata la fortuna cinquecentesca del testo bembiano: $\mathrm{cfr}$. T. Tasso, Rime I250 I-9, V. Colonna, Rime amorose disperse 8 I-4 (e L. Contile, Rime cristiane 47).

I4 Su questo nodo cruciale non ancora sciolto vd. ora Albonico, Appunti su "forma" e "materia", cit., e le conclusioni di Vagni in questo volume.

I5 Bembo, Rime, cit. 
Ch'ancor sento tornar pur come soglio madonna in quel suo atto dolce honesto ad acquetare il cor misero et mesto,

che pare un rimando valido solo per l'«at to honesto et piano», in cui in realtà emerge anche un altro luogo petrarchesco, $R v f$ I7O 4, «la mia nemica in atto humile et piano». L'attacco è in effetti tutto nel segno di Petrarca, giacché nel primo distico pare individuabile pure la scissione in due frasi di $R v f 80$ 4, «non pò molto lontan esser dal fine», combinata alla reminiscenza di $R v f$ 3645 , «Omai son stanco, et mia vita reprendo». È tuttavia significativo che nel complesso la quartina riassembli i vv. 9-Io del sonetto 62 dei Sonetti e canzoni, dedicato all'invocazione al sonno: «Sì bella e sì pietosa in vista umile / madonna apparve al cor doglioso e stanco». ${ }^{16}$ Bembo preleva da Sannazaro la condizione di affaticamento («Giaceami stanco» rielabora sulla base di Petrarca «al cor doglioso e stanco»), la visitazione della donna in sogno («madonna apparve» in identica posizione metrica) e uno degli elementi della dittologia che qualifica madonna («pietosa»), con l'eliminazione però dell'allusione a Cino (38 37-38, «Io la vidi sì bella e sì gentile / e di vista sì umìle»), confermata dalle varianti. Anche in questo caso peraltro la riscrittura segna un acquisto in precisione: Bembo non dice soltanto di essere stanco, ma di essere vicino alla morte, e la donna non solo appare, ma concede il proprio aiuto.

L'influenza della lirica sannazariana è avvertibile pure nel sonetto 89, composto probabilmente negli anni dieci, di certo prima del I52I-22:

Fortuna, che sì spesso indi mi svia, tolga a gli occhi, a gli orecchi il proprio obietto, e 'n parte le dolcezze mie distempre;

al cor non torrà mai l'alto diletto ch'ei prova di veder la donna mia ovunque io vado, et d'ascoltarla sempre.

Il punto di partenza è una delle rime disperse (I5 I-8):

Hai tolto agli occhi il suo beato obietto, invida Morte, et a la speme mia, togliendo con tua falce strana e ria colei ond'io prendevo ogni diletto.

Ma l'alto suo valor, che nel suo petto vive sempre e vivrà, ovonque sia, e l'unica beltate e leggiadria

I6 Il motivo dell'apparizione femminile in sogno è diffuso nella lirica aragonese, cfr. ad es. De Jennaro, Canzoniere 58 Io-I3. 
non curan né l'altrui n'il tuo dispetto.

Innanzitutto un'osservazione tematica: in questo caso non vi è una perfetta sovrapposizione, bensì uno slittamento semantico, poiché Bembo trasforma un testo in morte in uno di lontananza. Sannazaro denuncia la morte dell'amata (I quartina) affermando però la possibilità di conservare la virtù e la bellezza della donna nella memoria (II quartina); Bembo invece oppone alla lontananza dall'amata per le circostanze del caso (I terzina) la persistenza dell'immagine e della voce di lei nel proprio cuore (II terzina). La distribuzione della materia rispetto ai comparti metrici è dunque uguale malgrado lo spostamento nelle terzine nel sonetto di Bembo. Gli elementi comuni sono molti e lasciano pochi dubbi circa la conoscenza del testo da parte di Bembo e il grado d'intenzionalità: condivisione di due rime (-ia e -etto) e insieme ad esse di tre rimanti (obietto, diletto, mia, con identico referente nei primi due casi), ripresa della ripetizione variata del verbo togliere («tolga [...] non torrà»-«Hai tolto [...] togliendo»), arricchita dalla coppia di complementi che dipendono dal primo termine («agli occhi [...] et a la speme mia» diventa «a gli occhi, a gli orecchi» sotto l'influsso di Rvf 270 4I, «rendi agli occhi, agli orecchi il proprio obgetto»), dell'avverbio sempre nonché, con variatio, dell'aggettivo alto (con il precedente di Rvf 28I 7-8, «l'alto diletto / che Morte à tolto») e dell'emistichio «ovonque sia», il quale diviene «ovunque io vada», ossia è riferito al poeta, non più alla donna. Va infine notato che il poliptoto assume un valore "strutturale" nel sonetto bembiano, in quanto funge da connettivo tra le due unità metriche e di conseguenza tra l'assenza reale e la presenza nel ricordo.

Al di là di questi rapporti, che pertengono ai temi e alla struttura profonda dei testi, al loro schema inventivo e retorico-sintattico, vi sono innumerevoli prelievi più minuti, che riguardano il lessico, i sintagmi e le locuzioni. In questa sede si è tuttavia ritenuto più opportuno concentrarsi su nessi del primo tipo, che coinvolgono i testi a più livelli, giacché, come detto, si tratta di un aspetto perlopiù trascurato. Si è infatti persuasi che per giungere a comprendere la cultura e la lirica di questi poeti sia indispensabile assumere una prospettiva più vicina alla loro, ossia quella di letterati di formazione umanistica e di poeti bilingui, latini e volgari, dunque di uomini abituati a lavorare con zibaldoni e altri strumenti affini, i quali presuppongono un approccio in primis tematico. ${ }^{17}$ Infine, un'indagine di questo genere permette parimenti di apprezzare maggiormente le personalità poetiche degli autori, non appiattendole sul solo modello petrarchesco, e restituendo loro la giusta profondità.

amelia.juri@unil.ch

I7 Per questo aspetto vd. di nuovo le riflessioni di Albonico, Appunti su "forma" e "materia", cit. 